\title{
Long-term Improvement after the Athens Protocol for Advanced Keratoconus with Significant Ectasia Progression in the Fellow Eye
}

Jorge Augusto Siqueira, Luis Claudio Dias, Renata Siqueira, Bruno Valbon, Rodrigo Santos

Daniel Dawson, Renato Ambrósio Jr

\begin{abstract}
To describe the long-term result of the Athens protocol (custom topography-guided advanced surface ablation followed by riboflavin-UVA collagen crosslinking in the same day) for progressive advanced keratoconus in the left eye, while the fellow right eye first presented with relatively mild keratoconus that was treated with a more conservative therapeutic approach of continuous contact lenses use with the goal for ectasia stabilization. Five years after the Athens protocol, a regression of over $10 \mathrm{D}$ (diopters) was observed on the sagittal curvature with significant regularization of corneal shape and improvement of distance corrected visual acuity (DVCA) in the left eye. The right eye, which was treated more conservatively, had an over $12 \mathrm{D}$ increase on sagittal curvature along with significant distortion of the corneal shape and reduction of DCVA.
\end{abstract}

Keywords: Keratoconus, Athens protocol, Contact lens.

How to cite this article: Siqueira JA, Dias LC, Siqueira R, Valbon B, Santos R, Dawson D, Ambrósio Jr R. Longterm Improvement after the Athens Protocol for Advanced Keratoconus with Significant Ectasia Progression in the Fellow Eye. J Kerat Ect Cor Dis 2013;2(3):143-146.

Source of support: Nil

Conflict of interest: None declared

\section{INTRODUCTION}

Keratoconus is a progressive, bilateral ectatic disorder in which the cornea becomes irregular and cone-shaped. ${ }^{1}$ The ectatic condition is related to a progressive thinning and bulging due to chronic biomechanical failure of the corneal stroma. Corneal collagen crosslinking was developed in 1998 by Prof Seiler et al for augmenting corneal rigidity through the application of riboflavin (vitamin-B2) eye drops followed by ultraviolet A (UV-A) radiation. ${ }^{2}$

The application of customized topography-guided surface ablation has been reported in patients with stable or subclinical keratoconus. ${ }^{3-5}$ The Athens Protocol ${ }^{6}$ consists of the combination of same-day advanced custom surface ablation and riboflavin-UVA collagen crosslinking. ${ }^{7-9}$ The main goal of the procedure is to regularize the cornea, ${ }^{10}$ not to provide primary refractive correction, so that this approach is considered as therapeutic. ${ }^{11}$

Special contact lens fitting, including RGP (rigid gas permeable) contact lenses, represents the main conservative therapeutic approach for visual rehabilitation of ectatic corneal diseases and is responsible for over $50 \%$ of the cases. ${ }^{12,13}$ Contact lenses may provide the best possible visual acuity because it regulates the corneal surface along with the tear film. ${ }^{14,15}$ However, there is no evidence that contact lens stabilize or prevent ectasia progression, while there is some anecdotal evience that it may be associated with a higher risk of ectasia progression due to continuous microtrauma from chronic RGP contant lense use. ${ }^{16,17}$

\section{CASE REPORT}

A Caucasian 26 years old presented with a history of decreased vision in his left eye since 2007. On examination, uncorrected distance visual acuity (UDVA) was 20/60 of the right eye and $<20 / 400$ of the left eye. Distance best corrected vision (DCVA) with spectacles was 20/20 in the right eye, and 20/80 in the left eye. Fundus examination was within normal limits in both eyes. Goldmann applanation IOP was $10 \mathrm{~mm} \mathrm{Hg}$ in OD and $7 \mathrm{~mm} \mathrm{Hg}$ in OS. Goldmann-correlated IOP(IOPg), corneal-compensated intraocular pressure (IOPcc), Corneal Hysteresis (CH), and corneal resistance factor (CRF) from the Ocular Response Analyzer (ORA, Reichert, Buffalo, NY) were, respectively, 14.1, 11.4, 7.8 and $8.8 \mathrm{~mm} \mathrm{Hg}$ of the right eye and 11.7, 7.6, 6.1 and $8.1 \mathrm{~mm} \mathrm{Hg}$ of the left eye. Keratoconus was confirmed by Placido diskbased corneal topography (iTrace) and rotating Scheimpflug corneal tomography (Pentacam, Oculus, Wetzlar, Germany). Wavefront optimized manifest refraction ${ }^{18}$ with data from iTrace (Tracey Technologies, Houston, TX) was $-1.25-2.75 \times$ $56^{\circ}=20 / 20$ of the right eye and $-9.75-3.75 \times 1^{\circ}=20 / 60$ of the left eye. The patient was fitted with RGP contact lens, providing a best corrected visual acuity of 20/15 of the right eye and 20/30 of the left eye.

Six months later, the patient returned complaining of decreased vision of the left, which failed to improve with contact lens wear. Wavefront (WF) optimized manifest refraction was $-1.50-2.75 \times 49^{\circ}=20 / 20$ of the right eye, and $-11.75-6.00 \times 162^{\circ}=20 / 80$ of the left. Significant progression of ectasia was demonstrated of the left eye with an increase of the maximal keratometric value $\left(\mathrm{K}_{\max }\right)$ of $2.77 \mathrm{D}$, while the right eye remained stable. 
Considering the progressive nature of the ectasia, the intolerance to further contact lens wear, the amount of irregularity, and the thinnest corneal thickness value of $456 \mu \mathrm{m}$ of the left eye, the Athens Protocol ${ }^{6}$ was performed accordingly to the description by Kanellopoulos as an alternative for keratoplasty. Advanced surface ablation was performed with the Allegretto (Alcon-Wavelight, Earlagen, Germany). Epithelial removal was accomplished using the PTK (Phototherateutic keratectomy) mode set for 50 microns with a $6.5 \mathrm{~mm}$ optical zone and an $8.8 \mathrm{~mm}$ blend zone followed by subsequent same day custom topography-guided ablation (TCAT), based on Oculyzer data, which removed 47 microns of tissue in the deepest ablation zone. Mitomycin C $(0.005 \%)$ was applied for 45 seconds followed by copious irrigation with $50 \mathrm{cc}$ of cold $\left(4^{\circ} \mathrm{C}\right)$ BSS. Dextran-free $0.1 \%$ Riboflavin solution was instilled every 2 minutes for 30 minutes. The yellow flare was verified before UVA irradiation at $3 \mathrm{~mW} / \mathrm{cm}^{2}$ for 30 minutes was competed. A bandage soft contact lens (SofLens 66, Bausch \& Lomb Inc, Rocheste, New York) was placed until the cornea epithelialized on post operative day 6 with no adverse event besides moderate pain in the first three days after the procedure. One month after the procedure, UCVA was 20/100. The patient had a second opinion with another corneal subspecialist, who advised against corneal collagen crosslinking OD and who also indicated that continuous RGP contact lens wear in both eyes was necessary.

The patient returns in 2012 (4 years after the procedure) complaining of bad vision in both eyes and contact lens intolerance. UCVA was 20/100 of the right eye and 20/60 of the left eye. The wavefront (WF) optimized manifest refraction was $-13.50-7.00 \times 8^{\circ}=20 / 70$ of the right eye and $-11.50-1.00 \times 169^{\circ}=20 / 25$ of the left eye. Comparative

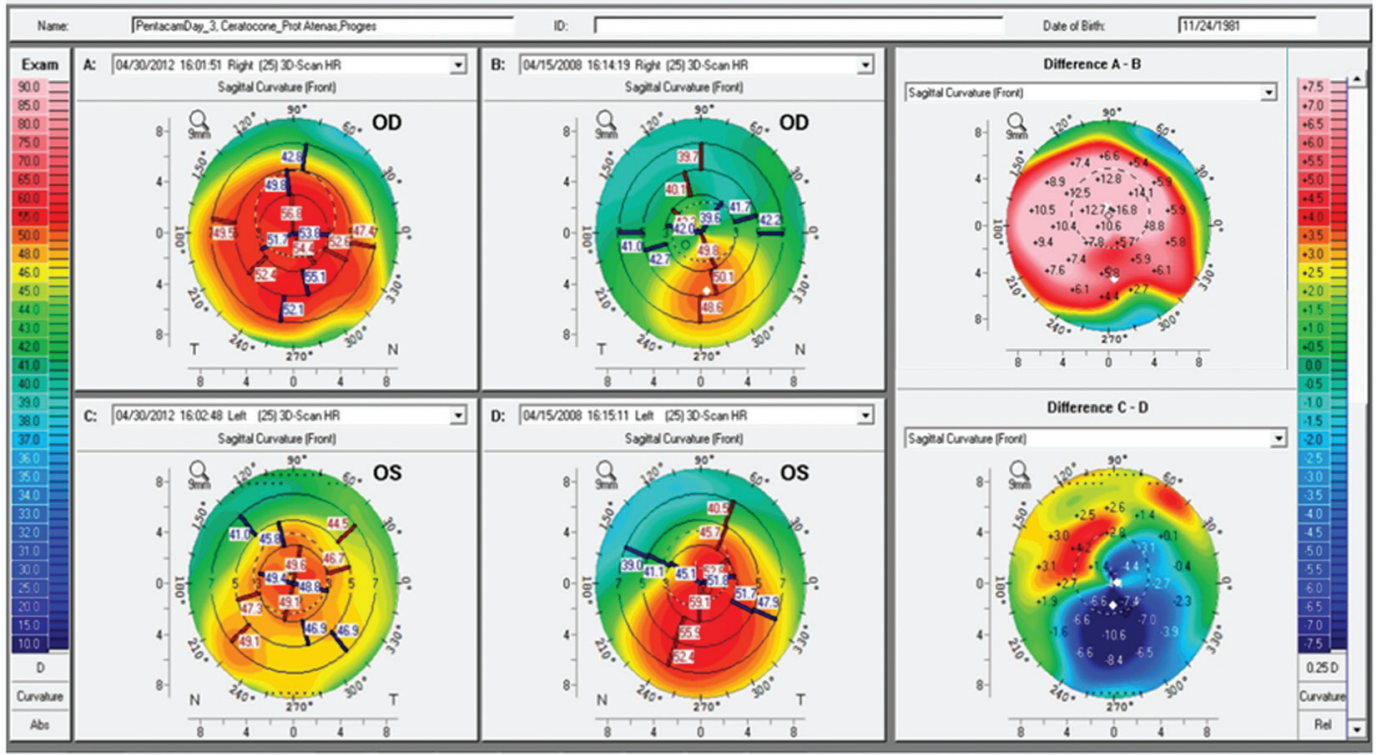

Fig. 1: Sagittal curvature differential maps

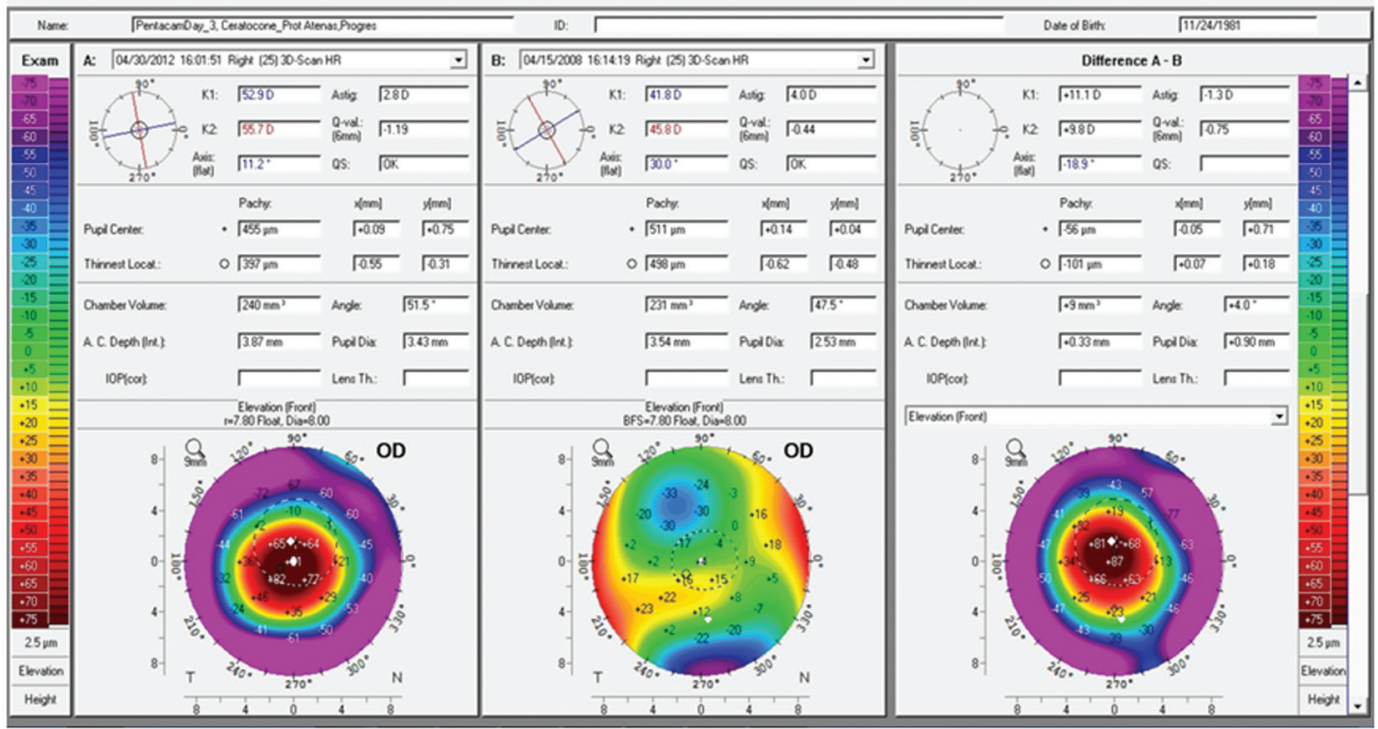

Fig. 2: Right eye: Maps of front elevation (A) postoperative, $(B)$ preoperative and the difference A-B, with the same BFS 


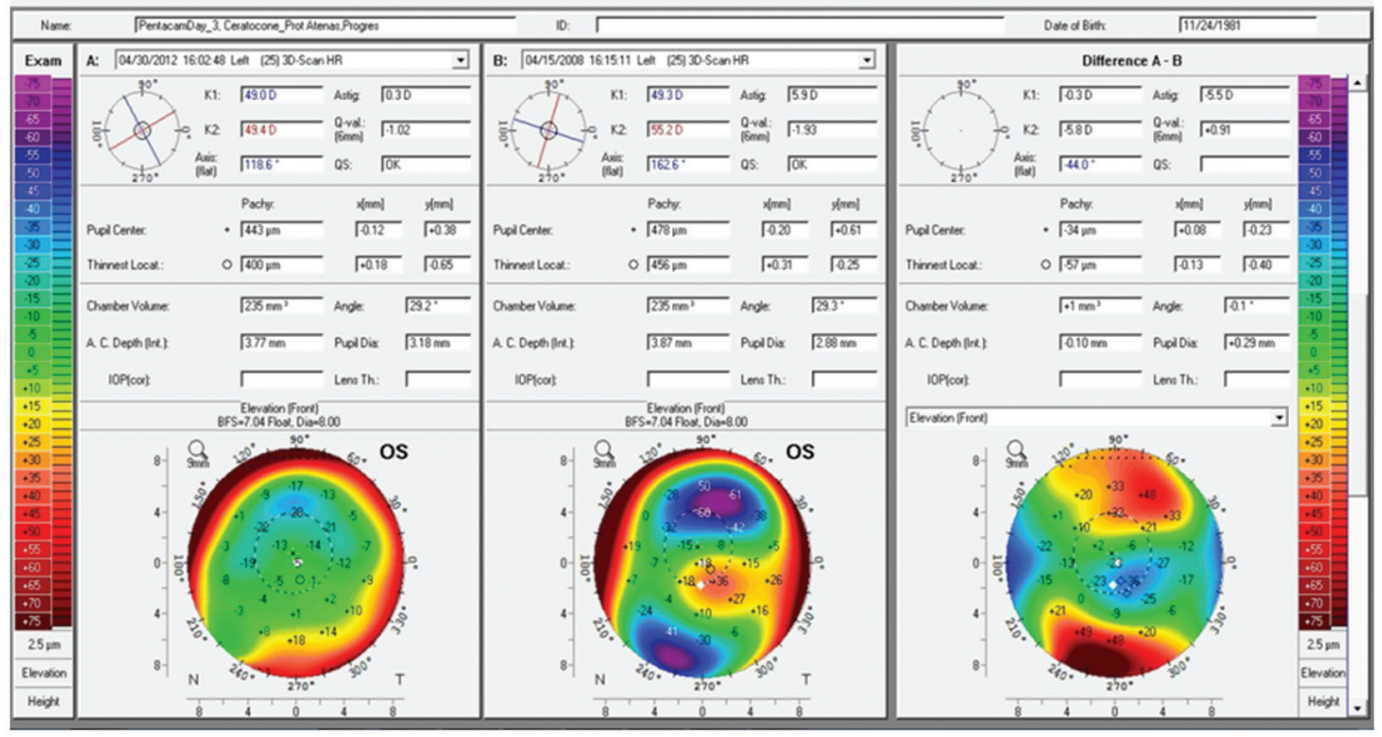

Fig. 3: Left eye: Maps of front elevation (A) postoperative, $(B)$ preoperative and the difference A-B, with the same BFS

subtraction front sagittal curvature maps from 2008 and 2012 are presented in Figure 1. Figures 2 and 3 include the elevation differential maps for OD and OS respectively. 12.6 $\mathrm{D}$ of corneal steepening is observed OD (Figs 1A and B) and 10.6 D of flattening is observed OS (Figs 1C and D).

Figure 2 provides the front elevation maps for OD in 2008 and 2012. The same reference was chosen of $7.8 \mathrm{~mm}$ as best fit sphere (BFS) for the first evaluation. Over 80 microns of increase in elevation is observed as well as a decrease in central corneal thickness from 511 to $455 \mu \mathrm{m}$ (at pupil center) and an increase in ACD (anterior chamber depth) from $3.54 \mathrm{~mm}$ to $3.87 \mathrm{~mm}$.

Figure 3 provides the front elevation maps for OS in 2008 and 2012. The same reference was chosen of $7.04 \mathrm{~mm}$ as best fit sphere (BFS) for the first evaluation. Thirty-six microns of decrease in elevation was observed and a decrease in central keratometric astigmatism from $5.9 \mathrm{D}$ to $0.3 \mathrm{D}$. A mild decrease in central corneal thickness from 478 to $443 \mu \mathrm{m}$ (at pupil center) and an decrease in ACD (anterior chamber depth) from 3.87 to $3.77 \mathrm{~mm}$.

The patient was advised about the need for therapeutic surgery for OD and surgical options, including the possibility of keratoplasty, ${ }^{19}$ and phakic IOL as a refractive option for OS.

\section{DISCUSSION}

The patient presented with advanced progressive keratoconus of the left eye, which was treated by the Athens protocol, and the right eye had a mild and relatively stable keratoconus presentation with good distance corrected visual acuity with spectacles. The patient had a second opinion, who advised against crosslinking of the right eye and to continuously wear RGP contact lens OD. This case illustrates that the use of contact lens does not stop the progression of keratoconus.
Moreover, continuous microtrauma related to continuous contact lens wear may be an important factor to accelerate ectasia progression in some patients. ${ }^{16,17}$ After 4 years, a regression of more than $10 \mathrm{D}$ of astigmatism of the left eye was associated with regularization of the corneal surface and improvement in the BCVA after the Athens protocol. This result is consistent with the literature. ${ }^{6,20-22}$

\section{CONCLUSION}

The Athens protocol is a therapeutic alternative for patients with progressive keratoconus to prevent or even delay keratoplasty. This is not a refractive procedure. Refractive correction with a phakic IOL becomes an possibility if best spectacle corrected vision is good. However, the progressive nature of ectatic corneal diseases should be considered and patient education is fundamental. Surgery for keratoconus should be indicated when visual rehabilitation is not possible with glasses or contact lenses and when there is documented or a significant risk of ectasia progression. While contact lenses provide the best possible treatment for the irregular astigmatism, there is no evidence to support the benefit of contact lens for decreasing the risk of ectasia progression. In fact, contact lens fitting should be considered with caution if spectacles provide adequate visual acuity.

\section{REFERENCES}

1. Rabinowitz YS. Keratoconus. Surv Ophthalmol 1998;42: 297-319.

2. Spoerl E, Huhle M, Seiler T. Induction of cross-links in corneal tissue. Exp Eye Res 1998;66:97-103.

3. Alpins N, Stamatelatos G. Customized photoastigmatic refractive keratectomy using combined topographic and refractive data for myopia and astigmatism in eyes with forme fruste and mild keratoconus. J Cataract Refract Surg 2007;33:591-602. 
4. Koller T, Iseli HP, Donitzky C, Ing D, Papadopoulos N, Seiler T. Topography-guided surface ablation for forme fruste keratoconus. Ophthalmology 2006;113:2198-2202.

5. Cennamo G, Intravaja A, Boccuzzi D, Marotta G. Treatment of keratoconus by topography-guided customized photorefractive keratectomy: two-year follow-up study. J Refract Surg 2008;24:145-149.

6. Kanellopoulos AJ. Comparison of sequential vs same-day simultaneous collagen crosslinking and topography-guided PRK for treatment of keratoconus. J Refract Surg 2009;25: S812-818.

7. Wasilewski D, Mello GH, Moreira H. Impact of collagen crosslinking on corneal sensitivity in keratoconus patients. Cornea 2012.

8. Viswanathan D, Males J. Prospective longitudinal study of corneal collagen crosslinking in progressive keratoconus. Clin Experiment Ophthalmol 2012.

9. Cheema AS, Mozayan A, Channa P. Corneal collagen crosslinking in refractive surgery. Curr Opin Ophthalmol 2012;23:251-256.

10. Oshika T, Tomidokoro A, Maruo K, Tokunaga T, Miyata N. Quantitative evaluation of irregular astigmatism by fourier series harmonic analysis of videokeratography data. Invest Ophthalmol Vis Sci 1998;39:705-709.

11. Ambrósio R Jr. Cirurgia refrativa terapêutica: por que diferenciar? Revista Brasileira de Oftalmologia 2013;72:85-86

12. Wagner H, Barr JT, Zadnik K. Collaborative longitudinal evaluation of keratoconus (CLEK) study: methods and findings to date. Cont Lens Anterior Eye 2007;30:223-232.

13. Weed KH, Macewen CJ, McGhee CN. The Dundee University Scottish Keratoconus Study II: a prospective study of optical and surgical correction. Ophthalmic Physiol Opt 2007;27: 561-567.

14. Mandathara PS, Fatima M, Taureen S, Dumpati S, Ali MH, Rathi V. RGP contact lens fitting in keratoconus using FITSCAN technology. Cont Lens Anterior Eye 2013;36:126-129.

15. Fatima T, Acharya MC, Mathur U, Barua P. Demographic profile and visual rehabilitation of patients with keratoconus attending contact lens clinic at a tertiary eye care centre. Cont Lens Anterior Eye 2010;33:19-22.

16. McMonnies CW. Mechanisms of rubbing-related corneal trauma in keratoconus. Cornea 2009;28:607-615.

17. Barr JT, Zadnik K, Wilson BS, et al. Factors associated with corneal scarring in the collaborative longitudinal evaluation of keratoconus (CLEK) study. Cornea 2000;19:501-507.

18. Ambrósio R Jr, Caldas DL, Silva RSD, Pimentel LN, Valbon BDF. Impacto da análise do 'wavefront' na refratometria de pacientes com ceratocone. Revista Brasileira de Oftalmologia 2010;69:294-300.
19. Vazirani J, Basu S. Keratoconus: current perspectives. Clin Ophthalmol 2013;7:2019-2030.

20. Kremer I, Aizenman I, Lichter H, Shayer S, Levinger S. Simultaneous wavefront-guided photorefractive keratectomy and corneal collagen crosslinking after intrastromal corneal ring segment implantation for keratoconus. J Cataract Refract Surg 2012;38:1802-1807.

21. Spadea L. Collagen crosslinking for ectasia following PRK performed in excimer laser-assisted keratoplasty for keratoconus. Eur J Ophthalmol 2012;22:274-277.

22. Kymionis GD, Grentzelos MA, Portaliou DM, et al. Photorefractive keratectomy followed by same-day corneal collagen crosslinking after intrastromal corneal ring segment implantation for pellucid marginal degeneration. J Cataract Refract Surg 2010;36:1783-1785.

\section{ABOUT THE AUTHORS}

\section{Jorge Augusto Siqueira (Corresponding Author)}

Eye Surgeon, Instituto de Olhos Renato Ambrósio, Rio de Janeiro Brazil, Phone: 552122644430, e-mail: jorgejass1@yahoo.com.br Research Associate, Rio de Janeiro Corneal Tomography and Biomechanics Study Group, Brazil

\section{Luis Claudio Dias}

Research Associate, Rio de Janeiro Corneal Tomography and Biomechanics Study Group, Brazil

\section{Renata Siqueira}

Research Associate, Rio de Janeiro Corneal Tomography and Biomechanics Study Group, Brazil

\section{Bruno Valbon}

Doctorate PhD Fellow, Federal University of São Paulo, Brazil

\section{Rodrigo Santos}

Refractive Surgery Fellow, Federal University of São Paulo, Brazil

\section{Daniel Dawson}

Assistant Professor, Department of Ophthalmology, University of Florida, Gainesville, USA

\section{Renato Ambrósio Jr}

Clinical Director of Cornea and Refractive Surgery, Instituto de Olhos Renato Ambrosis, Rio de Janeiro, Brazil; Associate Professor of Ophthalmology, Federal University of São Paulo, Brazil 